\title{
The Ambitious Goal of Validating Prognostic Biomarkers for Systemic Sclerosis-related Interstitial Lung Disease
}
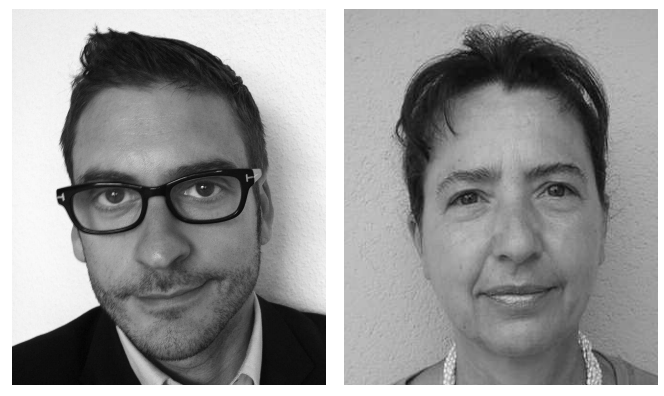

Interstitial lung disease (ILD) often complicates the course of systemic sclerosis ( $\mathrm{SSc}$ ), mainly in patients with the diffuse subset of disease and with anti-Scl70 antibody positivity ${ }^{1,2}$. Postmortem examination gives evidence that nearly all patients with SSc develop pulmonary fibrosis, although to a very different extent individually; high-resolution computed tomography (HRCT) shows interstitial lung involvement in about two-thirds of the cases ${ }^{2}$.

Together with pulmonary arterial hypertension, severe pulmonary fibrosis is the leading cause of SSc-related death, as demonstrated by Steen and Medsger among the very large Pittsburgh cohort ${ }^{3}$. These data were confirmed by a study of the SSc population included in the European League Against Rheumatism Scleroderma Trials and Research group database ${ }^{4}$. With regard to the course of ILD, some patients develop severe and rapidly progressive interstitial lung involvement during the early phase of the disease, while others may show an indolent course without a relevant influence on pulmonary function or survival ${ }^{1,5}$. Assessment of lung involvement and close monitoring of lung function are therefore mandatory.

During the last decade efforts have been undertaken to identify predictors of rapid deterioration in SSc lung interstitial involvement. The Scleroderma Lung Study (SLS) and the Genetics versus Environment in Scleroderma Outcome Study (GENISOS) assembled the largest cohorts for this purpose $^{6,7}$. These efforts were intended mainly to drive treatment choice, but also to avoid serial HRCT scans for patient followup to reduce radiation exposure. One of the most important findings was that, similar to idiopathic pulmonary fibrosis ${ }^{8}$, the decline in forced vital capacity (FVC) and DLCO turned out to mirror the degree of changes observed on $\mathrm{HRCT}^{9}$. An accelerated rate of decline in FVC is associated with poor survival ${ }^{7}$. Despite these attempts, at present there are no validated lung functional/radiological measures or serologic biomarkers that would allow prediction of the rate of progression of pulmonary fibrosis over time, and thus allow physicians to individualize the management for different patients' subtypes. An ideal biomarker should be noninvasive, reliable, and cheap. Many attempts have been made to find such a candidate biomarker for SSc-ILD, but data have come mostly from retrospective single-center studies ${ }^{10}$. We know now that there are a number of candidate serum proteins that correlate with the presence or severity of ILD in the context of SSc. The final step for the validation of a biomarker requires prospective, well-designed multicenter studies in heterogeneous populations. One major problem in the validation process is the identification of factors that influence the basal levels of these biomarkers, such as covariates, genetic assets, and baseline disease severity ${ }^{10}$.

For biologically complex diseases such as SSc, characterized by autoimmunity, fibrosis, and microvascular damage, the multiplicity of underlying pathogenetic patterns suggests that disease prediction may come to depend on combinatorial analysis of many mediators ${ }^{11,12}$. A lesson from rheumatoid arthritis (RA) is the multibiomarker disease activity (MBDA) score ${ }^{13}$. Recently, Centola, et al developed a quantitative serum-based measure of RA disease activity, based on 12 biomarkers from 130 that were screened, which was consistently associated with clinical disease activity levels and damage progression measured by radiography ${ }^{13}$. Hirata, et al confirmed in a Japanese cohort that the MBDA score reflects current clinical disease activity and can track changes in disease activity over time ${ }^{14}$. Although this recent model is encouraging, to date, the panel of investigated biomarkers for SSc-ILD is limited, compared with the panel of investigated biomarkers in idiopathic pulmonary fibrosis ${ }^{10}$. Evidence has been provided for the ability of serum KL-6, surfactant protein-D (SP-D), and CC-chemokine ligand 18 (CCL18) to correlate with the likelihood of progression of SSc-ILD and for monitoring treatment response ${ }^{10}$.

In this issue of The Journal, Elhaj, et al from the GENISOS cohort raise critical questions on the value of serum SP-D and CCL18 as prognostic biomarkers for

See Can serum SP-D or CCL18 predict outcome of ILD, page 1114 
SSc-ILD ${ }^{15}$. The authors investigated the predictive significance of these 2 pneumoproteins for disease outcome of SSc-ILD in a large, well-characterized multiethnic cohort of 266 patients with early SSc from the GENISOS cohort, using advanced statistical modeling that takes into account longitudinal data at each followup point. For 83 patients it was possible to collect complete followup for biomarkers over 4 years. This allowed the authors to include all data points and account for dependence between longitudinal FVC and survival, reducing the bias resulting from the fact that patients with more rapid decline in FVC are more likely to die. Although autoantibody subsets such as antitopoisomerase I (ATA) and anti-RNP subtypes ${ }^{10}$ have been found to be associated with fibrosis in SSc, the investigators chose serum CCL18 and SP-D for their lung specificity and the evidence coming from studies on idiopathic interstitial pneumonias ${ }^{10}$. While serum level of SP-D correlated with FVC obtained at the same time, and CCL18 was a predictor of short-term decline in FVC, the main finding of the study was that neither SP-D nor CCL18 was a longterm predictor of the course of FVC in patients with early SSc. Similarly, a composite score of SP-D and CCL18 was a significant predictor of short-term decline in FVC, but did not predict its longterm rate of decline $e^{15}$.

CCL18, previously known as pulmonary and activation-regulated chemokine (PARC), is constitutively expressed at high levels in the lungs, mainly by alveolar macrophages, and is chemotactic for a variety of mononuclear cells ${ }^{16}$. CCL18 can stimulate fibroblast collagen and has been found to be increased in serum and bronchoalveolar lavage (BAL) fluid in a number of fibrotic lung diseases, including SSc-ILD ${ }^{16}$. Three longitudinal studies with a total of 116 patients with SSc-ILD investigated the correlation of serum CCL18 with ILD activity determined by HRCT, lung function, and BAL analysis, with promising results ${ }^{16,17,18}$. Tiev, et al analyzed serum CCL18 levels in 83 SSc patients with ILD over a 4-year followup period ${ }^{18}$. At a cutoff level of $187 \mathrm{ng} / \mathrm{ml}$ for identifying subsequent worsening of lung disease within 2 years or death, serum CCL18 was independently predictive of worsening ILD $(\mathrm{HR}=5.4 ; \mathrm{p}=0001)^{18}$. Similar results have been published by Prasse, et al from studies of patients with idiopathic pulmonary fibrosis ${ }^{19}$. The study from Elhaj, et al was intended to confirm the reliability of serum CCL18 as a longterm predictor of ILD course, but the results were negative ${ }^{15}$. In contrast to the studies of Tiev, et al and Prasse, et al, the current study did not show a significant predictive significance of baseline serum CCL18 for longterm change in FVC in both univariate and multivariate analysis. The huge differences in the results of the longitudinal analyses are probably due not only to the study design (a large heterogeneous multicenter cohort) and the more complex statistical approach that verified the predictive value of the biomarker over time, but also to the fact that changes of CCL18 in serum may not reflect its local production in the fibrotic lung in SSc. This point needs further investigation.

With regard to SP-D, a lipoprotein secreted by type II alveolar epithelial cells and airway Clara cells, together with SP-A, this pneumoprotein is involved in the maintenance of alveolar surface tension and the host defense system in the lung, and has been found to be elevated in patients with SSc-ILD, correlating with degree of fibrosis and pulmonary function test results 6,20 . Elhaj, et al found no difference in the predictive quality of baseline or longitudinal SP-D in serum compared to CCL18 separately as well as combined in a score. The only difference was that, in the multivariate analysis, SP-D remained an independent correlate of concomitant FVC. Also, a subgroup analysis based on disease type (limited/diffuse) or ATA positivity did not yield significant results for the short-term or longterm predictive value of SP-D or CCL18.

A remarkable subanalysis from Elhaj, et al attempted to elucidate the effects of immunosuppression on the usefulness of SP-D and CCL18 as biomarkers for SSc-ILD. The investigators conducted the same statistical analyses as for the overall group in the patients treated with immunosuppressive agents at the baseline visit, $32 \%$ of the entire cohort, excluding those receiving hydroxychloroquine or low-dose prednisone. The results substantially confirmed findings in the overall group, except for stronger evidence that combining serum CCL18 and SP-D could be of some value for predicting short-term decline in FVC. This approach, separately analyzing the patients treated with immunosuppressants, can also provide additional information for the reliability of these serum proteins as treatment response markers in randomized controlled trials.

The utility of 2 serum pneumoproteins, SP-D and CCL18, for longterm prediction of SSc-ILD disease course, but not for assessment of disease severity, has been questioned in this study. The new exploratory approach based on joint analysis of longitudinal measurements of serum biomarkers, sequential pulmonary function test results, and survival provides a new instrument to verify through large, prospective validation studies - the results of single-center studies, which tend to draw premature and over-enthusiastic conclusions on the longterm prognostic value of such biomarkers.

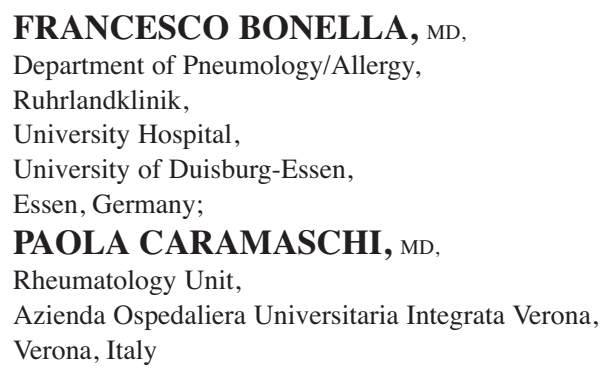


Address correspondence to Dr. F. Bonella, Department of Pneumology and Allergy, Ruhrlandklinik, University Hospital, University of Duisburg-Essen, Tüschener Weg 40, 45239 Essen, Germany.

E-mail: francesco.bonella@ruhrlandklinik.uk-essen.de

\section{REFERENCES}

1. Olson AL, Brown KK, Fischer A. Connective tissue diseaseassociated lung disease. Immunol Allergy Clin North Am 2012;32:513-36.

2. Launay D, Remy-Jardin M, Michon-Pasturel U, Mastora I, Hachulla E, Lambert M, et al. High resolution computed tomography in fibrosing alveolitis associated with systemic sclerosis. J Rheumatol 2006;33:1789-801.

3. Steen VD, Medsger TA. Changes in causes of death in systemic sclerosis, 1972-2002. Ann Rheum Dis 2007;66:940-4.

4. Tyndall AJ, Bannert B, Vonk M, Airo P, Cozzi F, Carreira PE, et al. Causes and risk factors for death in systemic sclerosis: A study from the EULAR Scleroderma Trials and Research (EUSTAR) database. Ann Rheum Dis 2010;69:1809-15.

5. Fischer A, Swigris JJ, Groshong SD, Cool CD, Sahin H, Lynch DA, et al. Clinically significant interstitial lung disease in limited scleroderma: Histopathology, clinical features, and survival. Chest 2008;134:601-5.

6. Hant FN, Ludwicka-Bradley A, Wang HJ, Li N, Elashoff R, Tashkin DP, et al. Surfactant protein D and KL-6 as serum biomarkers of interstitial lung disease in patients with scleroderma. J Rheumatol 2009;36:773-80.

7. Assassi S, Sharif R, Lasky RE, McNearney TA, Estrada YM, Draeger $\mathrm{H}$, et al. Predictors of interstitial lung disease in early systemic sclerosis: A prospective longitudinal study of the GENISOS cohort. Arthritis Res Ther 2010;12:R166.

8. du Bois RM, Nathan SD, Richeldi L, Schwarz MI, Noble PW. Idiopathic pulmonary fibrosis: Lung function is a clinically meaningful endpoint for phase III trials. Am J Respir Crit Care Med 2012;186:712-5.

9. Moore OA, Goh N, Corte T, Rouse H, Hennessy O, Thakkar V, et al. Extent of disease on high-resolution computed tomography lung is a predictor of decline and mortality in systemic sclerosis-related interstitial lung disease. Rheumatology 2013;52:155-60.
10. Lota HK, Renzoni EA. Circulating biomarkers of interstitial lung disease in systemic sclerosis. Int J Rheumatol 2012;2012:121439.

11. Murray LA, Rubinowitz A, Herzog EL. Interstitial lung disease: Is interstitial lung disease the same as scleroderma lung disease? Curr Opin Rheumatol 2012;24:656-62.

12. Baraut J, Michel L, Verrecchia F, Farge D. Relationship between cytokine profiles and clinical outcomes in patients with systemic sclerosis. Autoimmun Rev 2010;10:65-73.

13. Centola M, Cavet G, Shen Y, Ramanujan S, Knowlton N, Swan KA, et al. Development of a multi-biomarker disease activity test for rheumatoid arthritis. PLoS One 2013;8:e60635

14. Hirata S, Dirven L, Shen Y, Centola M, Cavet G, Lems WF, et al. A multi-biomarker score measures rheumatoid arthritis disease activity in the BeSt study. Rheumatology 2013 Apr 17. [Epub ahead of print]

15. Elhaj M, Charles J, Pedroza C, Liu X, Zhou X, Estrada YM, et al. Can serum surfactant protein D or CC-chemokine ligand 18 predict outcome of interstitial lung disease in patients with early systemic sclerosis? J Rheumatol 2013;40:1114-20.

16. Prasse A, Pechkovsky DV, Toews GB, Schafer M, Eggeling S, Ludwig C, et al. CCL18 as an indicator of pulmonary fibrotic activity in idiopathic interstitial pneumonias and systemic sclerosis. Arthritis Rheum 2007;56:1685-93.

17. Kodera M, Hasegawa M, Komura K, Yanaba K, Takehara K, Sato S. Serum pulmonary and activation-regulated chemokine/CCL18 levels in patients with systemic sclerosis: A sensitive indicator of active pulmonary fibrosis. Arthritis Rheum 2005;52:2889-96.

18. Tiev KP, Hua-Huy T, Kettaneh A, Gain M, Duong-Quy S, Toledano $\mathrm{C}$, et al. Serum CC chemokine ligand-18 predicts lung disease worsening in systemic sclerosis. Eur Respir J 2011;38:1355-60.

19. Prasse A, Probst C, Bargagli E, Zissel G, Toews GB, Flaherty KR, et al. Serum CC-chemokine ligand 18 concentration predicts outcome in idiopathic pulmonary fibrosis. Am J Respir Crit Care Med 2009;179:717-23.

20. Bonella F, Volpe A, Caramaschi P, Nava C, Ferrari P, Schenk K, et al. Surfactant protein D and KL-6 serum levels in systemic sclerosis: Correlation with lung and systemic involvement. Sarcoidosis Vasc Diffuse Lung Dis 2011;28:27-33.

J Rheumatol 2013;40:1034-6; doi:10.3899/jrheum.130549 